\title{
Comparative dimensional tomographic study of the mandibular condyle of individuals with transverse maxillary deficiency
}

\author{
Andréa Guedes Barreto Gonçales ${ }^{1}$, Victor Tieghi Neto², Eduardo Sanches Gonçales ${ }^{3}$, Ana Lúcia Álvares Capelozza ${ }^{4}$ \\ 'DDS, MsC. Stomatology Department. São Paulo University. Bauru Dental School \\ ${ }^{2}$ DDS, PhD. Stomatology Department. São Paulo University. Bauru Dental School \\ ${ }^{3} \mathrm{DDS}$, PhD. Associated Professor of Oral and Maxillofacial Surgery. Stomatology Department. São Paulo University. Bauru Dental School \\ ${ }^{4} \mathrm{DDS}$, PhD. Full Professor of Radiology. Stomatology Department. São Paulo University. Bauru Dental School
}

\begin{abstract}
Transverse maxillary deficiency is characterized by posterior uni or bilateral crossbite, crowded and rotated teeth, as well as high palate. Its treatment in adult individuals is surgically assisted rapid palatal expansion. The aim of this study was to verify the occurrence of dimensional alterations in the mandibular condyles of patients with TMD submitted to surgically assisted maxillary expansion. Measurements of the mandibular condyles using the DISTANCE tool in cone beam computed tomography iCat software were performed. The values obtained were submitted to statistical analysis by the paired t-test and the results showed statistically significant dimensional reduction in the axial posterior-anterior lateral $(-0.74 \mathrm{~mm})$, axial posterior-anterior lateral left $(-0.90 \mathrm{~mm})$ and coronal medium right $(-1.24 \mathrm{~mm})$ dimensions. The coronal inferior $(1.13 \mathrm{~mm})$, coronal inferior left $(1.78 \mathrm{~mm})$ and coronal superior-inferior right $(0.76 \mathrm{~mm})$ measurements showed statistically significant dimensional increase. The results allowed us to conclude that dimensional alterations occurred in the mandibular condyles in individuals with maxillary transversal deficiency that underwent surgically assisted rapid palatal expansion (SAPE), which can be understood by remodeling, since they are characterized by dimensional increase or reduction, depending on the location where the measurement was performed.
\end{abstract}

Keywords: Computed tomography. Malocclusion. Mandibular condyle.

\section{Introduction}

The dento-facial skeletal deformities can be defined as a defect of conformation of an organ or body part and result from changes in the growth and development of the bones of the face, especially maxilla and mandible. However other structures, organs and systems related to bones involved may be affected, causing problems in positioning of teeth, dental arches, masticatory function, phonation, swallowing, temporomandibular joints, breathing and facial aesthetics ${ }^{1}$. Transverse maxillary deficiency (TMD) is characterized by posterior uni or bilateral crossbite, crowded and rotated teeth, as well as high palate ${ }^{2,3}$.

There are three basic alternatives for the treatment of TMD: orthopedic maxillary expansion, surgically assisted palatal expansion (SAPE) and Le Fort I 
segmental osteotomy. SAPE is indicated for the treatment of subjects with TMD larger than $5 \mathrm{~mm}$, orthodontic/orthopedic expansion failure, need of expansion greater than $7 \mathrm{~mm}$ and for individuals with TMD and skeletal age of 15 years or more ${ }^{2}$, The treatment of TMD in individuals with skeletal maturity is surgically assisted palatal expansion (SAPE) and this treatment has shown good results4, even with the description of different surgical techniques ${ }^{2}$.

The effects of SAPE are observed not only in the dental $\operatorname{arch}^{5}$, but also in the adjacent structures ${ }^{6-10}$ and mandibular dental $\operatorname{arch}^{11}$. The procedure is not free of complications, such as the occurrence of pain in the temporomandibular joint (TMJ) in $3 \%$ of the cases, mobility (3\%) and loss (3\%) of the upper central incisors, sinusitis $(6 \%)$, paresthesia $(3 \%)$, bleeding $(3 \%)$, external resorption of teeth $(3 \%)$ and displacement and tilt of the teeth $(3 \%)^{12}$.

Cone beam computed tomography (CBCT) has been used in the planning and follow up of oral and maxillofacial surgeries, allowing the comparison of bone changes ${ }^{13}$. Since SAPE exerts effects on the adjacent maxillary structures, like changes in occlusion during this procedure, possible pain and changes in the TMJ, and that CBCT images allow the visualization and measurements of bony structures, the aim of the present study was to verify the occurrence of dimensional changes in mandibular condyles of individuals with TMD that underwent SAPE.

\section{Material and methods}

In the present study, measurements were done by the use of CBCT of the mandibular condyles. For this, preoperative and postoperative measurements $(\mathrm{mm})$ of mandibular condyle of 14 individuals that underwent SAPE were done. It was approved by the Research Ethics Committee of the Bauru School of Dentistry of São Paulo University (Protocol no. 1702011/11-30-2011). In axial view, measurements of the lateral-medial, posterior-anterior lateral, posterior-anterior central and posterior-anterior medial sizes of the articular surface were performed. In the coronal reformats, the superior lateral-medial, inferior lateral-medial, medium lateral-medial and superior-inferior sizes were measured.

All scans were performed on an i-CAT Classic (Imaging Science International, Hatfield, Pennsylvania, USA) using the following protocol: 0.3mm voxel and Extended Height 20/20sec. The CBCTs were filed on CD and the linear measurement tool (DISTANCE) of the i-Cat Vision program was used for obtaining the measurements as described below.

On the i-Cat Vision homepage, we choose the name of the individual and open the CBCT by double clicking on the individual name. After the CBCT loads, the Screen MPR option was selected, obtaining the sagittal, axial and coronal views (Figures 1, 2 and 3).

The standardization of the condyle positions was obtained as follows: the mandible image was positioned on sagittal reformatting showing the articular cavity, articular eminence, mandibular angle, ramus and condyle. In this image, we positioned the red reference line (corresponding to axial reformatting) at the lower point of the articular eminence surface. The blue reference line (corresponding to the coronal reformatting) was positioned in the center of the condyle, perpendicular to the red reference line. The Distance tool was used to determine the center of the condyle by the measurement of the distance from the posterior to the anterior portion of the condyle surface on the red reference line. The condyle center corresponded to half the distance measured on the red reference line (Figure 1) and all the measures done in the axial and coronal views (figures 2 and 3) were made with this standardization.

The measures done on axial reformatting are shown in Figure 2: a) the lateral-medial distance of the articular surface measured between the lateral and medial condyle poles (axial lateral-medial), b) posterior-anterior lateral size of the condyle articular surface measured in the lateral portion of the condyle, c) posterior-anterior central size of the condyle articular surface measured in the central portion of the condyle, d) posterior-anterior medial size of the condyle articular surface measured in the medial portion of the condyle.

For the determination of the sites to be measured, the distance between the lateral and medial poles of the condyles was initially measured (axial lateral-medial measurement) on axial reformatting. The center of this measure determined the position of the axial posterior-anterior central measurement, which was measured perpendicular to the external surfaces. Similarly, the axial posterior-anterior lateral and medial distances were measured, since its position corresponded to half the distance between the condyle center (axial posterior-anterior central measurement) and the lateral and medial poles, respectively (Figure 2).

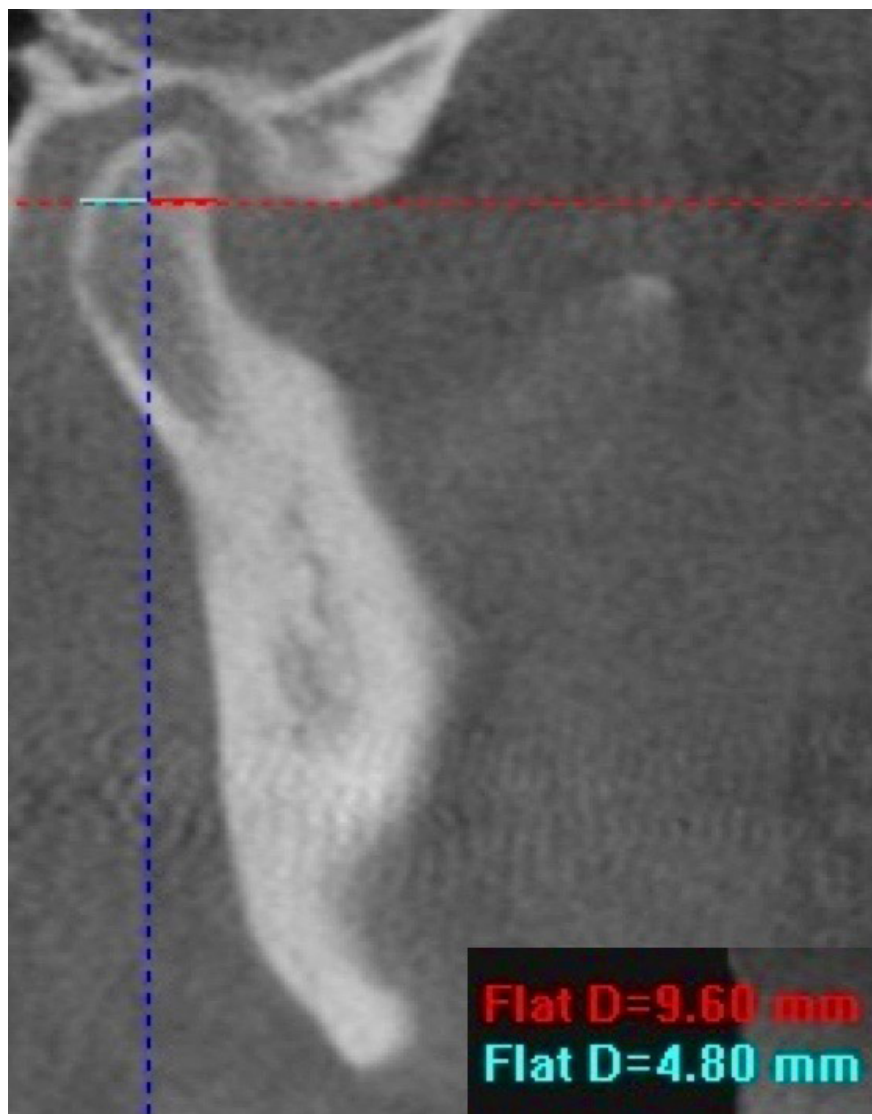

Fig.1. Standardization of the positioning in sagittal reformatting. 


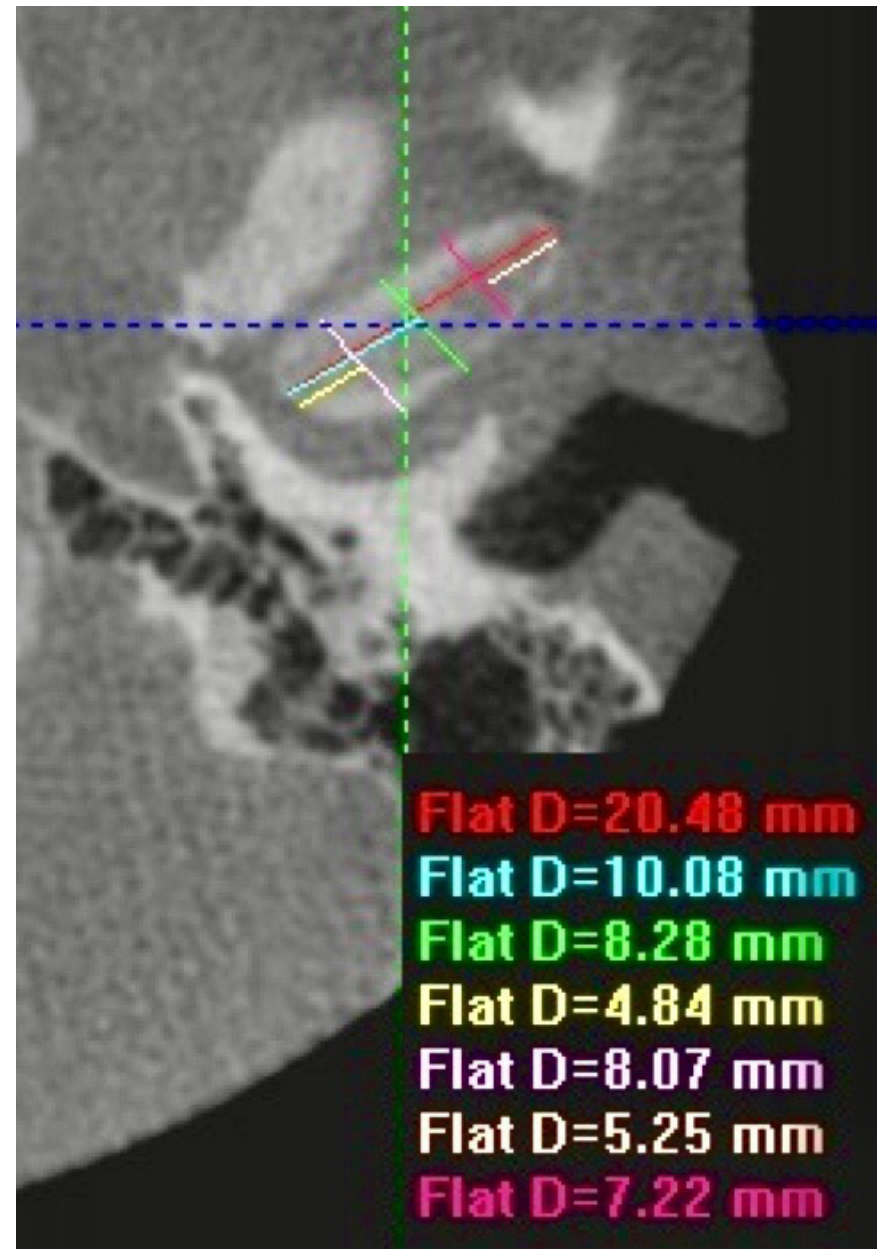

Fig.2. Axial measurements: lateral-medial (red), posterior-anterior central (green), posterior-anterior lateral (pink) and posterior-anterior medial (light pink), carried out in the condyles. The other measures that appear (yellow, light blue and salmon) were performed to determine the locations of the axial measurements.

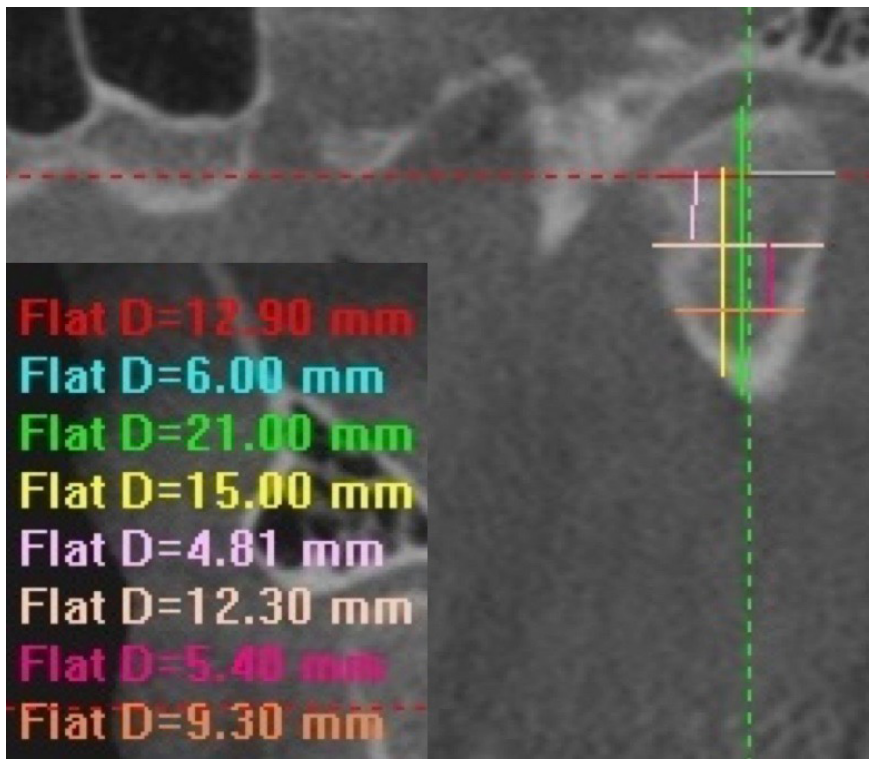

Fig.3. Coronal Superior measurements (red), Coronal Superior-inferior measurement (green), Coronal Medium measurement (salmon) and Coronal Inferior measurement (orange), carried out in the condyles on coronal reformatting. The other measures that appear (yellow, light pink, pink and light blue) were performed to determine the locations of the coronal measures.
The following measurements were performed on coronal reformatting: a) the lateral-medial superior size, measured from the lateral to medial surface at the bottom of the condyle joint surface (Coronal superior measurement), b) medium medial-lateral size, measured from lateral to the medial, in the intermediate portion of the condyle, equidistant from the upper and lower measures, c) inferior medial-lateral size, measured from the lateral to medial in the lower portion of the condyle, and d) superior-inferior measure, taken from the articular surface of the condyle center until the lower cortical bone (Figure 3).

In a similar way implemented in axial reformatting, on coronal reformatting the locations to be measured were standardized as follows: the distance between the lateral and medial poles of the condyle were measured at the lower bound of the articular surface and this was the superior coronal measurement. At the center of this measurement, perpendicular to the outer surface of the articular surface, from upper to lower, the Coronal Superior-inferior measurement was done. The lower bound of this measure was the intersection of the central measurement line with the external cortical of the condyle. The distance between the Coronal Superior measurement and the lower limit of the condyle was then measured. It was then divided into three and the Coronal medium measurement was measured in the lateral-medial sense in the upper third of the measure, while the Coronal Inferior measurement was performed in the lower third, also in the medial-lateral direction (Figure 3).

All measurements were obtained in preoperative and postoperative CBCTs, tabulated and submitted to statistical analysis by paired $t$ test. After 15 days of the completion of the first data collection ${ }^{14}$, all measurements were redone to determine the random and systematic error by the Intraclass Correlation Coefficient (ICC) $)^{15}$.

\section{Results}

We used 28 CBCTs from fourteen individuals with TMD, submitted to SAPE. Five of the individuals were men and nine were women, with an average age of 24.28 years (DP 4.39). Six individuals $(42.86 \%)$ used the Hass expander device while $8(57.15 \%)$ used the Hyrax expander device. The average postoperative period was 192 days (SD 16.03).

Table 1 shows the average (A), standard deviation (SD), difference between preoperative and postoperative averages (dif), paired t-test result (p), when the right and left condyles were considered together, while Table 2 represents the average (A), standard deviation (SD), difference between preoperative and postoperative averages (dif), paired t-test result (p), when the right and left condyles were considered separately.

In general, it was observed that there were changes in dimensional mandibular condyles, characterized by increase or decrease, depending on the site of the measure. The analysis of the condyles together (per individual) was shown to be similar to the analysis of the isolated condyles. Thus, the results of the measures showed an increase in the axial lateral-medial $(0.19 \mathrm{~mm})$, axial posterior-anterior central $(0.15 \mathrm{~mm})$, coronal superior $(0.27 \mathrm{~mm})$, coronal inferior $(1.13 \mathrm{~mm})$ and coronal 
superior-inferior $(0.48 \mathrm{~mm})$ measures. The reduction of the measures was observed at the axial posterior-anterior lateral $(-0.74 \mathrm{~mm})$, axial posterior-anterior medial $(-0.35 \mathrm{~mm})$ and coronal medium $(-0.28 \mathrm{~mm})$ measures. Only the axial posterioranterior lateral and coronal inferior measures were statistically significant (Table 1).

Table 1 - Average (A), standard deviation (SD), difference between preoperative (Preop) and postoperative (Postop) averages (dif), paired t-test result $(\mathrm{p})$, when the right and left condyles were considered together (same individual) $\left({ }^{*} \mathrm{p}<0.05\right)$.

\begin{tabular}{ccccccc}
\hline Measure & Preop & \multicolumn{7}{c}{ Postop } & & \\
\hline & $\mathrm{A}$ & $\mathrm{SD}$ & $\mathrm{A}$ & $\mathrm{SD}$ & dif. & $\mathrm{p}$ \\
\hline Axial Lateral-medial & 18.32 & 2.51 & 18.51 & 2.53 & 0.19 & 0.372 \\
\hline $\begin{array}{c}\text { Axial Posterior-anterior } \\
\text { lateral }\end{array}$ & 7.05 & 1.15 & 6.31 & 1.02 & -0.74 & $0.001^{*}$ \\
\hline $\begin{array}{c}\text { Axial Posterior-anterior } \\
\text { central }\end{array}$ & 7.32 & 1.36 & 7.47 & 1.49 & 0.15 & 0.487 \\
\hline $\begin{array}{c}\text { Axial Posterior-anterior } \\
\text { medial }\end{array}$ & 6.86 & 1.15 & 6.51 & 1.33 & -0.35 & 0.147 \\
\hline Coronal Superior & 14.87 & 2.48 & 15.14 & 2.76 & 0.27 & 0.320 \\
\hline Coronal Medium & 13.62 & 2.53 & 13.33 & 2.85 & -0.28 & 0.410 \\
\hline Coronal Inferior & 7.25 & 1.21 & 8.37 & 1.78 & 1.13 & $0.000^{*}$ \\
\hline Coronal Superior - inferior & 18.58 & 3.25 & 19.06 & 3.66 & 0.48 & 0.090 \\
\hline
\end{tabular}

Table 2 - Average (A), standard deviation (SD), difference between preoperative (Preop) and postoperative (Postop) averages (dif), paired t-test result $(\mathrm{p})$, when the right and left condyles were considered separate $(* \mathrm{p}<0.05) . \mathrm{R}=$ right. $\mathrm{L}=\mathrm{Left}$.

\begin{tabular}{|c|c|c|c|c|c|c|}
\hline Measure & Preop & & Postop & & & \\
\hline & A & SD & A & SD & dif. & $p$ \\
\hline Axial Lateral-medial $R$ & 18.61 & 2.51 & 18.65 & 2.64 & 0.05 & 0.860 \\
\hline Axial Lateral-medial L & 18.03 & 2.56 & 18.37 & 2.51 & 0.34 & 0.344 \\
\hline $\begin{array}{c}\text { Axial Posterior-anterior } \\
\text { lateral } \mathrm{R}\end{array}$ & 6.61 & 1.18 & 6.03 & 0.97 & -0.58 & 0.094 \\
\hline $\begin{array}{c}\text { Axial Posterior-anterior } \\
\text { lateral } \mathrm{L}\end{array}$ & 7.49 & 0.98 & 6.59 & 1.02 & -0.90 & $0.005^{*}$ \\
\hline $\begin{array}{c}\text { Axial Posterior-anterior } \\
\text { central } \mathrm{R}\end{array}$ & 6.89 & 1.36 & 7.31 & 1.67 & 0.42 & 0.303 \\
\hline $\begin{array}{c}\text { Axial Posterior-anterior } \\
\text { central L }\end{array}$ & 7.74 & 1.27 & 7.63 & 1.33 & -0.11 & 0.513 \\
\hline $\begin{array}{c}\text { Axial Posterior-anterior } \\
\text { medial R }\end{array}$ & 6.69 & 1.51 & 6.65 & 1.43 & -0.05 & 0.889 \\
\hline $\begin{array}{c}\text { Axial Posterior-anterior } \\
\text { medial L }\end{array}$ & 7.02 & 0.63 & 6.37 & 1.26 & -0.65 & 0.061 \\
\hline Coronal Superior R & 14.54 & 2.72 & 14.39 & 2.70 & -0.15 & 0.621 \\
\hline Coronal Superior L & 15.21 & 2.27 & 15.89 & 2.70 & 0.68 & 0.121 \\
\hline Coronal Medium R & 13.54 & 2.91 & 12.30 & 3.00 & -1.24 & $0.000^{*}$ \\
\hline Coronal Medium L & 13.69 & 2.19 & 14.36 & 2.36 & 0.67 & 0.234 \\
\hline Coronal Inferior R & 7.07 & 1.38 & 7.54 & 1.70 & 0.47 & 0.123 \\
\hline Coronal Superior L & 7.42 & 1.04 & 9.20 & 1.48 & 1.78 & $0.000^{*}$ \\
\hline $\begin{array}{c}\text { Coronal Superior-infe- } \\
\text { rior R } \\
\end{array}$ & 18.16 & 3.69 & 18.92 & 3.88 & 0.76 & $0.007^{*}$ \\
\hline $\begin{array}{c}\text { Coronal Superior-infe- } \\
\text { rior L }\end{array}$ & 19.00 & 2.81 & 19.20 & 3.58 & 0.20 & 0.694 \\
\hline
\end{tabular}

When analyzed separately, each condyle showed an increase in axial lateral-medial right $(0.05 \mathrm{~mm})$ and left $(0.34 \mathrm{~mm})$, axial posterior-anterior central right $(0.62 \mathrm{~mm})$, axial posterioranterior central right $(0.42 \mathrm{~mm})$, coronal superior left $(0.67$ $\mathrm{mm})$, coronal inferior right $(0.47 \mathrm{~mm})$ and left $(1.78 \mathrm{~mm})$ and coronal superior-inferior right $(0.76 \mathrm{~mm})$ and left $(0.20 \mathrm{~mm})$ measures. The isolated analysis of each condyle allowed us to also see a reduction in axial dimensions of the posterior-anterior lateral right $(-0.58 \mathrm{~mm})$ and left $(-0.90 \mathrm{~mm})$, posterior-anterior central left $(-0.11 \mathrm{~mm})$, posterior-anterior medial right $(-0.05$ $\mathrm{mm})$ and left $(-0.65 \mathrm{~mm})$, coronal superior right $(-0.15 \mathrm{~mm})$ and coronal medium right $(-1.24 \mathrm{~mm})$. When we analyzed the condyles separately, apart from the axial posterior-anterior lateral left, coronal medium right, coronal superior left and coronal superior-inferior right measures (table 2), these values were not statistically significant.

For error analysis, the ICC was used; all measures were carried out by the same examiner and respecting the time of no less than 15 days. The ICC was excellent, since all their values were greater than 0.75 .

\section{Discussion}

In individuals who have reached skeletal maturity due to the consolidation of craniofacial sutures ${ }^{16}$, the TMD treatment is orthodontic and surgical and the surgical step is known as Surgically Assisted Maxillary Expansion (SAPE) ${ }^{2,3}$. After the surgical procedure, maxillary expansion itself is accomplished by gradual, periodic and successive activation of the expander device that results in distribution of forces through the maxillas and adjacencies, which lead to the separation and enlargement of them ${ }^{17,18}$.

The occurrence of TMJ pain was evidenced in 3\% of the cases of individuals subjected to $\mathrm{SAPE}^{12}$, which may be considered a low incidence, and the incidence of other complications was also low, even though those that occurred more often reached a maximum of $6 \%$ (sinusitis) ${ }^{12}$. The incidence of TMJ pain allow us to believe that the anatomical configuration of the human face, especially the relationship between the maxilla and mandible, TMJs, muscles of mastication and the dental occlusion may lead to the occurrence of SAPE effects in the mandible. It should be noted that Albuquerque et al. ${ }^{12}$ (2013) considered TMJ pain in a generic form, perhaps not only including isolated pain in the TMJ, but also muscle aches. Since the mandibular condyle is one of the components of TMJ, by the factors described above, it is subjected to the effects of SAPE. This study was a retrospective study that evaluated only the preoperative and postoperative CBCT of the individuals submitted to SAPE. Thus there are no conditions to discuss about signs and symptoms or even about clinical features as Albuquerque et al.12 (2013) did.

Our results corroborate the aforementioned because there were changes in the dimensional mandibular condyles, characterized by increase or decrease, based in the preoperative and postoperative measurements carried out, depending on the measure. Such changes could have taken place, for example, by the occlusal overload generated by premature dental contacts, leading to changes of mandibular position and eventually in the condyle remodeling. 
However, even when an increase in dimensions of axial measures occurs, when separately analyzing each condyle, only the axial posterior-anterior lateral left, coronal medium right, coronal superior left and coronal superior-inferior right measurements were statistically significant $\left({ }^{*} p<0.05\right)$. Such fact might demonstrate that the remodeling occurs preferentially in certain areas and these areas may vary according to the occlusal interference and because of this, they are asymmetrical. It is expected that where there was a statistically significant decrease of the measures, the condyle was reabsorbed and a statistically significant increase occurred because of bone formation.

The TMJ is a bilateral joint in which both sides work simultaneously, and therefore we also evaluated the condyles in sets. Similarly, only the axial posterior-anterior lateral and coronal inferior measures were statistically significant. It is believed that, even when the individual is considered, the occlusal interference might be responsible for possible asymmetric condyle remodeling.

Although studies ${ }^{19-21}$ showed that occlusal forces can affect the condyle morphology and may lead to condyle remodeling and CBCT was a useful tool for monitoring condylar bone changes, it should be noted that the statistical result found can be due the number of studied subjects or the methodology employed. This study did not verify the occurrence of occlusal changes and premature contacts, as well as the presence or absence of symptoms related to TMJs or masticatory muscles. Thus, one can only infer the occurrences just described.

With regard to the method employed, the applicability of CBCT in Dentistry appears to be questionable because some studies $^{19,22-26}$ claimed that the CBCT was a reliable and accurate method for verification of changes and carry out measures in TMJ as a whole and specifically in the mandibular condyles. This is especially true when the sagittal and coronal views are considered, being efficient in the determination of changes related to the positioning and shape of the condyle and joint surfaces ${ }^{27,28}$.

It is believed that cited studies, corroborated the methodology used, since they correlated CBCT with dimensional and positioning and shape changes of condyles. Despite having difficulties in detection by the $\mathrm{CBCT}$ of the condylar defect smaller than $2 \mathrm{~mm}^{29,30}$, this difficulty would not be expected to occur in the present study, since the distances representing the condylar dimensions were measured, but not bone defects. t. In addition, the ICC15 was excellent, confirming the accuracy of the measurements performed.

Thus, based on the methodology used and the sample studied, it seems justified to conclude that in individuals with TMD submitted to SAPE, dimensional changes occur in the mandibular condyles, perhaps by remodeling, since they were characterized by an increase and reduction of the condylar dimensions.

\section{References}

1. Assis DSFR, Duarte MA, Gonçales ES. Clinical evaluation of the alar base width of patients submitted to surgically assisted maxillary expansion. Oral Maxillofac Surg. 2010 Sep;14(3):149-54. doi: 10.1007/s10006-0100211-3.

2. Betts NJ, Vanarsdall RL, Barber HD, Higgins-Barber K, Fonseca RJ. Diagnosis and treatment of transverse maxillary deficiency. Int J Adult
Orthodon Orthognath Surg. 1995;10(2):75-96.

3. Gonçales ES, Polido WD. Surgical and orthodontic treatment of transverse maxillary deficiency: concepts for the oral and maxillofacial surgeon and a case report. Rev Inst Cienc Saude. 1998;16(1):55-9. Portuguese.

4. Gonçales ES, Assis DR, Capelozza ALA, Alvares LC. Indirect digital radiographic study of the effect of surgically assisted palatal expansion (SAPE) on the nasal septum. Rev Dent Press Ortod Ortop Facial. 2007;12(5):85-91. Portuguese.

5. Garib DC, Henriques JFC, Janson G, Freitas MR, Fernandes AY. Periodontal effects of rapid maxillary expansion with tooth-tissue-borne and tooth-borne expanders: A computedtomography evaluation. Am J Orthod Dentofacial Orthop. 2006 Jun;129(6):749-58.

6. Assis DSFR, Ribeiro Júnior PD, Duarte MAH, Gonçales ES. Evaluation of the mesio-buccal gingival sulcus depth of the upper central incisors in patients submitted to surgically assisted maxillary expansion. Oral Maxillofac Surg. 2011 Jun;15(2):79-84. doi: 10.1007/s10006-010-0233-x.

7. Babacan H, Sokucu O, Doruk C, Ay S. Rapid maxillary expansion and surgically assisted rapid maxillary expansion effects on nasal volume. Angle Orthod. 2006 Jan; 76(1):66-71.

8. Compadretti GC, Tasca I, Bonetti GA. Nasal airway measurements in children treated by rapid maxillary expansion. Am J Rhinol. $2006 \mathrm{Jul}-$ Aug;20(4):385-93.

9. Koudstaal MJ, Poort LJ, van der Wal KGH, Wolvius EB, Prahl-Andresen $B$, Schulten AJM. Surgically assisted rapid maxillary expansion (SARME): a review of the literature. Int J Oral Maxillofac Surg. 2005 Oct;34(7):70914.

10. Gurgel JA, Malmström, MFV, Pinzan-Vercelino CR. Ossification of the midpalatal suture after surgically assisted rapid maxillary expansion. Eur J Orthod. 2012 Feb;34(1):39-43. doi: 10.1093/ejo/cjq153.

11. McNamara JA. Maxillary transversal deficiency. Am J Orthod Dentofacial Orthop. 2000 May;117(5):567-70.

12. Albuquerque GC, Gonçales AGB, Tieghi Neto V, Nogueira AS, de Assis DSFR, Gonçales ES. Complications following surgically assisted palatal expansion. Rev Odontol UNESP. 2013;42(1):20-4. Portuguese.

13. Pereira-Filho VA, Monnazzi MS, Gabrielli MA, Spin-Neto R, Watanabe ER, Gimenez CM, et al. Volumetric upper airway assessment in patients with transverse maxillary deficiency after surgically assisted rapid maxillary expansion. Int J Oral Maxillofac Surg. 2014 May;43(5):581-6. doi: 10.1016/j.ijom.2013.11.002.

14. Houston WJB. The analysis of errors in orthodontic measurements. Am J Orthod. 1983 May;83(5):382-90.

15. Fleiss JL. Analysis of data from multiclinic trials. Control Clin Trials. 1986 Dec;7(4):267-75.

16. Ennes JP; Consolaro A. Median palatine suture: evaluation of degree of ossification in human skulls. Rev Dental Press Ortodon Ortop Facial. 2004:9(5):64-73. Portuguese.

17. Assis DS, Xavier TA, Noritomi PY, Gonçales ES. Finite element analysis of bone stress after SAPE. J Oral Maxillofac Surg. 2014 Jan;72(1):167. e1-7. doi: 10.1016/j.joms.2013.06.210.

18. Assis DS, Xavier TA, Noritomi PY, Gonçales AG, Ferreira O Jr, de Carvalho PC, Get al. Finite element analysis of stress distribution in anchor teeth in surgically assisted rapid palatal expansion. Int J Oral Maxillofac Surg. 2013 Sep;42(9):1093-9. doi: 10.1016/j.ijom.2013.03.024.

19. Kurusua A; Horiuchib M; Soma K. Relationship between Occlusal Force and Mandibular Condyle Morphology Evaluated by Limited Cone-Beam Computed Tomography Angle Orthod. 2009 Nov;79(6):1063-9. doi: 10.2319/120908-620R.1.

20. Liu M, Chen H, Yap AUJ, Fu K. Condylar remodeling accompanying splint therapy: a cone-beam computerized tomography study of patients with temporomandibular joint disk displacement. Oral Surg Oral Med Oral Pathol Oral Radiol. 2012 Aug;114(2):259-65. doi: 10.1016/j. 0000.2012.03.004. 
21. Pontual MLA, Freire JSL, Barbosa JMN, Frazão MAG, Pontual AA, Silveira MMF. Evaluation of bone changes in the temporomandibular joint using cone beam CT Dentomaxillofac Radiol. 2012 Jan;41(1):24-9. doi: $10.1259 / \mathrm{dmfr} / 17815139$.

22. Honey OB; Scarfe WC; Hilgers MJ; Klueber K; Silveira AM; Haskell BS; Farmang AG. Accuracy of cone-beam computed tomography imaging of the temporomandibular joint: Comparisons with panoramic radiology and linear tomography. Am J Orthod Dentofacial Orthop. 2007 Oct;132(4):429-38.

23. Ludlow JB; Laster WS; See M; Bailey LJ; Hershey HG; Hill C. Accuracy of measurements of mandibular anatomy in cone beam computed tomography images. Oral Surg Oral Med Oral Pathol Oral Radiol Endod. 2007 Apr;103(4):534-42.

24. Marques AP, Perrella A, Arita ES, Pereira MSF, Cavalcanti MG. Assessment of simulated mandibular condyle bone lesions by cone beam computed tomography. Braz Oral Res. 2010 Oct-Dec;24(4):467-74.

25. Moreira CR, Sales MAO, Lopes PML, Cavalcanti GP. Assessment of linear and angular measurements on three dimensional cone-beam computed tomographic images. Oral Surg Oral Med Oral Pathol Oral Radiol Endod.
2009 Sep;108(3):430-6. doi: 10.1016/j.tripleo.2009.01.032.

26. Zain-Alabdeen EH, Alsadhan RI. A comparative study of accuracy of detection of surface osseous changes in the temporomandibular joint using multidetector CT and cone beam CT. Dentomaxillofac Radiol. 2012 Mar;41(3):185-91. doi: 10.1259/dmfr/24985981.

27. Accorsi M, Velasco L. 3D diagnosis in orthodontics: the cone-beam tomography applied. Nova Odessa, São Paulo: Napoleão; 2011. Portuguese.

28. Bayrama M, Kayipmazb S, Sezginb OS, Küc M. Volumetric analysis of the mandibular condyle using cone beam computed tomography. Eur J Radiol. 2012 Aug;81(8):1812-6. doi: 10.1016/j.ejrad.2011.04.070.

29. Bastos LC, Campos PSF, Ramos-Perez FMM, Pontual AA, Almeida $S M$. Evaluation of condyle defects using different reconstruction protocols of cone-beam computed tomography. Braz Oral Res. 2013 Nov-Dec;27(6):503-9. doi: 10.1590/S1806-83242013000600010.

30. Patel A, Tee BC, Fields H, Jones E, Chaudhry J, Sun Z. Evaluation of cone-beam computed tomography in the diagnosis of simulated small osseous defects in the mandibular condyle. Am J Orthod Dentofacial Orthop. 2014 Feb;145(2):143-56. doi: 10.1016/j.ajodo.2013.10.014. 